\title{
On Laminar Flow of Non-Newtonian Fluids in Porous Media
}

\author{
Hassan E. Fayed* Nadeem A. Sheikh ${ }^{\dagger}$ and Oleg Iliev ${ }^{\ddagger}$ \\ Numerical Porous Media Center- \\ King Abdullah University of Science and Technology, Thuwal, Kingdom of Saudi Arabia \\ Dept. Flows and Materials Simulation- \\ Fraunhofer (ITWM) Kaiserslautern, Germany
}

October 4, 2015

\begin{abstract}
Flow of generalized Newtonian fluids in porous media can be modeled as a bundle of capillary tubes or a pore-scale network. In general, both approaches rely on the solution of Hagen-Poiseuille equation using power law to estimate the variations in the fluid viscosity due to the applied shear rate. Despite the effectiveness and simplicity, power law tends to provide unrealistic values for the effective viscosity especially in the limits of zero and infinite shear rates. Here instead of using power law, Carreau model [5] is used to determine the effective viscosity as a function of the shear strain rate. Carreau model can predict accurately variation of the viscosity at all shear rates and provide more accurate solution for the flow physics in a single pore. Using the results for a single pore, normalized Fanning friction coefficient has been calculated and plotted as a function of the newly defined Reynolds number based on pressure gradient. For laminar flow, the variation of the friction coefficient with Reynolds number has been plotted and scaled. It is observed that generalized Newtonian fluid flows show Newtonian nature up to a certain Reynolds number. At high Reynolds number deviation from the Newtonian behavior is observed. The main contribution of this paper is to present a closed form solution for the flow in a single pore using Carreau model, which allows for fast evaluation of the relationship between flux and pressure gradient in an arbitrary pore diameter. In this way we believe that our development will open the perspectives for using Carreau models in pore network simulations at low computational costs to obtain more accurate prediction for generalize Newtonian fluid flows in porous media.
\end{abstract}

\footnotetext{
${ }^{*}$ Corresponding author: Postdoctoral Fellow, email: hehady@vt.edu

${ }^{\dagger}$ Postdoctoral Fellow, email: ndahmed@gmail.com

‡Professor, email: iliev@itwm.fraunhofer.de
} 


\section{Introduction}

Non-Newtonian flow in porous media is important for many applications, such as heavy oil recovery, enchanced oil recovery, many classes of liquid polymer moulding, certain hygienic materials, etc. In non-Newtonian flows, shear stress is non-linearly related to the shear strain rate. In other words, apparent viscosity of the flow is a function of the shear rate. Non-Newtonian fluids are classified into three main categories,

- Time independent or generalized Newtonian fluids

- Time dependent fluids

- Visco-elastic Fluids

In this paper we are interested in modeling laminar flow of generalized Newtonian fluid in porous media. Pore scale simulations, similar to the case of Newtonian flow in porous media, from one side allow to gain understanding about the flow details, and from another side allow to compute macroscopic characteristics, such as permeability. Pore scale flow of generalized Newtonian fluids in porous media can be simulated by direct numerical simulation on 3D CT images, or by simulations on simplified geometries, such as pore network or bundle of tubes [21]. 3D simulation on CT images is a challenging task due to the complexity of the pore architecture and in the case of upscaling, due to the fact that often large volumes need to be considered in order to meet the requirement for a representative elementary volume. In the pore network approach the porous media is represented as a 3D network of pipes. Flow rate and pressure drop in each branch is determined to find the bulk flow rate through this network [12], [1], [3] and [18].

Pore network methods provide feasible alternative to the direct 3D simulation, from one side accounting for the complexity of the geometry, and from another side working with simple geometry, such as pore-bodies and pore-throats. A number of papers are dedicated to using pore network approach for simulation of generalized Newtonian flow in porous media, a representative set of such papers can be found in Table 1 below.

As it can be seen from the Table, most of the papers use the so called power law. Power law model has a big advantage which is the easiness of obtaining an analytical solution for a pipe flow that significantly accelerates the computations in the pore network algorithms. Despite the simplicity, power law tends to provide unrealistic values for the effective viscosity especially in the limits of zero and infinite shear rates. Having in mind that large and small flow rates through pore throats can co-exist in a heterogeneous porous media, we are seeking for an approach which will provide better accuracy, but at the same time will not lead to an essential increase of the computational costs. It is known that Carreau model [5] can predict accurately variation of the viscosity and thus can provide a more accurate solution for the flow physics in a single pore. The main contribution of this paper is to present a closed form

solution for the the flow of Carreau fluid in a pipe, which allows for fast evaluation of the ratio between flux and pressure gradient. Using the results for a single pore, normalized Fanning friction coefficient can be calculated and plotted as a function of the newly defined Reynolds number based on pressure gradient. For the laminar flow, the improved chart provides direct variation of friction coefficient at a given pressure gradient and/or average flow rate for any 
Table 1: Summary of previous work

\begin{tabular}{|c|c|c|}
\hline Reference & Configuration & Viscosity model \\
\hline Sabiri and Jacques [19] & $\begin{array}{l}\text { Packed bed represented by } \\
\text { collection of capillaries }\end{array}$ & Power law \\
\hline Lopez et al [12] & Network modeling & Truncated power law \\
\hline Sullivan et al $[22]$ & $\begin{array}{l}\text { 3D randomly generated } \\
\text { porous media }\end{array}$ & Power law \\
\hline Tosco et al [25] & $\begin{array}{l}\text { Synthetic two-dimensional } \\
\text { porous media }\end{array}$ & $\begin{array}{l}\text { Cross, Ellis and Carreau } \\
\text { models }\end{array}$ \\
\hline Yun [29] & Fractal porous media & Power law \\
\hline Balhoff et al [1] & Network modeling & Power law and Ellis model \\
\hline Tang and Lu [23] & Packed bed & Power law \\
\hline Di Federico et al [7] & $\begin{array}{l}\text { Randomly heterogeneous } \\
\text { porous domain }\end{array}$ & Power law \\
\hline Perrin et al [16] & Pore scale network model & $\begin{array}{l}\text { Power law and Carreau } \\
\text { model }\end{array}$ \\
\hline Morais et al. [14] & $\begin{array}{l}3 \mathrm{D} \text { realization of the Swiss- } \\
\text { Cheese model }\end{array}$ & $\begin{array}{l}\text { Power law and Herschel- } \\
\text { Bulkley model }\end{array}$ \\
\hline Liu and Masliyah [11] & $\begin{array}{l}\text { Packed bed and network of } \\
\text { flow straits }\end{array}$ & Cross model \\
\hline Metzner [13] & Pipe flow & Power law \\
\hline Liu and Masliyah [10] & $\begin{array}{l}\text { Ducts and Porous medium } \\
\text { matrix }\end{array}$ & $\begin{array}{l}\text { Power law, Cross and } \\
\text { Herschel-Bulkley model }\end{array}$ \\
\hline Tsakiroglou [26] & Pore network modeling & Meter and power law \\
\hline Woudberg [27] & $\begin{array}{l}\text { Rectangular representative } \\
\text { cell }\end{array}$ & Power law \\
\hline Guzel et al [9] & Poiseuille pipe flow & Power law \\
\hline Yao et al [28] & Poiseuille pipe flow & Power law \\
\hline Pakdemirli [15] & Poiseuille pipe flow & Power law \\
\hline
\end{tabular}


pore size. In this way we believe that our development will open the perspectives for using Carreau models in pore network simulation, allowing at law computational costs to obtain more accurate prediction for a broad class of porous media flows.

In the laminar flow region, generalized Newtonian fluids behaves like Newtonian fluids as long as shear rate has no significant effects on viscosity . At higher shear rates, viscosity changes at some critical shear rate and flow deviate from the Newtonian behavior to the non-Newtonian behavior. While at very high shear rates, the apparent viscosity tends to be constant and named as 'infinite-shear rate viscosity' $\mu_{\infty}$. The two apparent viscosities (constant in the limit of zero and infinite shear rate) are properties of the generalized Newtonian fluids [24]. To define an appropriate Reynolds number of a non-Newtonian fluid flows in a porous medium, the selection of a value for the viscosity is not straight forward. Therefore Reynolds number at which flow changes its behavior from quasi-Newtonian to nonNewtonian depends on the rheological parameters of the generalized Newtonian fluid [24]. Different rheological models have been developed to determine the viscosity of generalized Newtonian fluids at different shear rates such as power law, Carreau model, Cross model [5], [2]. All these models use different parameters to fit the experimental data. Carreau model provides better predictions of the viscosity compared to the power law. However, Carreau model can not be used to develop analytical solution. Instead a numerical technique is used to determine the friction coefficient as a function of pressure gradient for non-Newtonian fluids.

Friction coefficient is a function of the applied shear stress at the pipe walls which is a non-linear function of the strain rate in the case of non-Newtonian fluid flow. Shear rate and hence viscosity are not known at the pipe walls. Pressure drop due to walls shear stress can be estimated if we know friction coefficient. Dodge and Metzner [6] used fanning friction factor $f$ to model laminar pipe flow for non-Newtonian fluids. Their work relied on power law model and dimensional analysis. Because of the varying viscosity from the pipe center line to the walls, Dodge and Metzner [6] developed a generalized Reynolds number and is given by

$$
R e_{D M}=\frac{\rho D^{n} \bar{W}^{2-n}}{K\left(\frac{3 n+1}{4 n}\right)^{n} 8^{n-1}}
$$

where $K$ is the consistency factor and $n$ is the index in the viscosity power law and $\bar{W}$ denotes for the mean axial velocity. This Reynolds number will be used later in the results section as $R e_{D M}$ and friction coefficient versus $R e_{D M}$ will be presented.

Similarly Guzel [9] defined a new Reynolds number $R e_{G}$ obtained from averaging of local Re across the pipe cross sectional area. Experimental work have also been conducted using different non-Newtonian fluids to determine friction factor at different $R e_{G}$. Guzel [9] plotted Moody diagram for Glycerin, Carbopol and Xanthan solutions based on Reynolds number $R e_{G}$. In his experiment, friction factor of the non-Newtonian fluids is slightly less below the Newtonian theoretical curve $f=64 / R e_{G}$. While for turbulent flow different semitheoretical models for friction coefficient of non-Newtonian flows are summarized by Garcia [8].

Here we aim to present an accurate solution of the generalized Newtonian fluid flow in a circular capillary representing a pore throat. The normalized solution is obtained irrespective 
of the physical dimensions of the pore. Carreau viscosity model [5] has been used in this work. It is a realistic model and can predict correct profile of viscosity at all values of shear rates. However it is not possible to develop analytical relationship for pressure drop and flow rate in a single pore using Carreau viscosity model. Instead an iterative method is employed to solve the problem. Using the results of the flow velocity and pressure gradient, calculations of normalized fanning friction coefficient for a generalized non-Newtonian fluid flow are also preformed. The results are presented using a revised Reynolds number based on pressure gradient.

The organization of the paper is as follows. Mathematical model is presented in the section (2). Section (3) presents the results and discussion of friction coefficient. In the end conclusions are presented in section (4).

\section{Mathematical Model}

In this section an incompressible, laminar fully developed isothermal axisymmetric flow of generalized Newtonian fluids through a circular pipe is assumed. We used cylindrical coordinates $\left(r^{*}, z^{*}\right)$, where $r^{*}$ is the radial coordinate and $z^{*}$ is the axial coordinate. Based on these assumptions, swirling velocity component is neglected. From the continuity equation and the no-penetration boundary condition at the pipe walls, we can deduce that radial velocity component is negligible for a fully developed pipe flow. Only axial velocity component $w^{*}$ is a function of the pipe radius.

The flow physics are described by Navier-Stokes equation with a shear rate dependent viscosity. According to the given assumptions, momentum balance in the axial direction is written as

$$
\frac{d p^{*}}{d z^{*}}=-\frac{1}{r^{*}} \frac{d}{d r^{*}}\left(\mu r^{*} \frac{d w^{*}}{d r^{*}}\right),
$$

where, $p^{*}=P^{*}-\rho g_{z}$ is the reduced pressure term and has units of $P a, r^{*}$ is the radius in meters and $w^{*}$ is the axial velocity and has units of $m / s . \mathrm{Eq}$ (2) has been simplified more by non-dimensionalization. In Newtonian incompressible flows, reference length and reference time are enough for the non-dimensionalization of the governing equations. For generalized Newtonian incompressible flow, reference length, reference time and reference mass are needed as basic variables for the non-dimensionalization of the above equation. Pipe radius $a$, zero-shear viscosity $\mu_{o}$ and reference velocity $w_{o}$ are chosen as reference variables. $\mathrm{Eq}(2)$ can be written in the non-dimensional form as

$$
\frac{d}{d r}\left[D r \frac{d w}{d r}\right]=-4 r,
$$

where $r=\frac{r^{*}}{a}, D=\frac{\mu}{\mu_{o}}$ and $w=\frac{w}{w_{o}}$. The non-dimensionalization of the z-momentum Eq results in the following expression of reference velocity $w_{o}$

$$
w_{o}=-\frac{a^{2}}{4 \mu_{o}} \frac{d p^{*}}{d z^{*}} .
$$


It is clear that values of $w_{o}$ depends on the applied pressure gradient. For the case of Newtonian flow $n=1, w_{o}$ is the local velocity at the pipe center line.

$\mathrm{Eq}(3)$ is subject to the following boundary conditions

$$
w(a)=0,
$$

and

$$
\frac{d w}{d r}=0
$$

Integrating Eq (3) and applying boundary condition in Eq 6 (zero shear rate at the pipe center line) results in the following equation

$$
\frac{d w}{d r}=\frac{-2 r}{D}
$$

This equation is nonlinear where the non-dimensional viscosity $D$ is a function of the nondimensional shear rate $\frac{d w}{d r}$ as given by Carreau model [5]. The model has two parameter that can be adjusted to fit the experimental data of the viscosity.

$$
D=\left[1+\left(\frac{w_{o} \lambda}{a} \frac{d w}{d r}\right)^{2}\right]^{\frac{n-1}{2}}
$$

BY Substitution from Eq (7) into Eq (8), we obtain the following algebraic expression for $D$

$$
D^{\frac{2 n}{n-1}}-D^{2}-4\left(\frac{w_{o} \lambda r}{2}\right)^{2}=0 .
$$

At each radius $r$, an iterative bisection method is used to find the roots of Eq (9). For shear thinning flows we used initial guess $D_{1 o}=1, D_{2 o}=0$ and for shear thickening flows we used $D_{1 o}=1, D_{2 o}=3$ for better convergence. The velocity profile is obtained by integrating $\mathrm{Eq}$ (7) for different viscosity parameters.

$$
w(r)=\int_{0}^{1} \frac{-2 r}{D} d r
$$

Trapezoidal rule is used to evaluate this integral as given by

$$
w_{i}=w_{i+1}+\Delta r\left[\frac{r_{i}}{D_{i}}+\frac{r_{i+1}}{D_{i+1}}\right]
$$

where $\Delta r=\frac{a}{N-1}, r_{i}=(i-1) \Delta r$ and $i=N-1, N-2, \ldots ., 1$ and the no slip boundary condition can be written as $w(N)=0$. Velocity profiles for different viscosity model parameters and flow rates under different pressure gradient are presented in the next section. 


\section{$3 \quad$ Results and Discussions}

Laminar Flows of Newtonian fluids in circular pipes are well understood. Usually pressure drop can be estimated for laminar as well as turbulent flows at different Reynolds number using Moody chart ( [20]) and/or other theoretical models for friction coefficient ( [9] [17], [8], [6]). For Newtonian fluids, molecular viscosity does not depend on the shear rate at a given pressure and temperature, while viscosity of non-Newtonian fluids depends on the shear rate. Moreover, shear rate is a non-linear function of the applied pressure gradient as given by $\mathrm{Eq}(2)$. Correct estimation of the viscosity is important to obtain accurate prediction of the flow rates due to certain pressure drop. Because of the sensitive nature of the power law, the results can vary drastically especially at low shear rates. Carreau viscosity model [5] on the other hand predicts realistic viscosity in the limit of very low and very high shear rate. However, Carreau model can not be used to develop analytical solutions for Eq 2.

The $1 \mathrm{D}$ model presented in this paper has been validated with the $3 \mathrm{D}$ results using FLUENT software. The same Carreau model presented here has been used in the 3D simulation by implementing a user defined function. In FLUENT simulation, parabolic velocity profile has been specified at the inlet and zero-pressure at the outlet boundary condition. The effective pressure gradient from the 3D simulation has been determined and used as an input to the 1D model. Velocity profiles from the 1D and 3D models have excellent agreement as shown in Figure 1a.

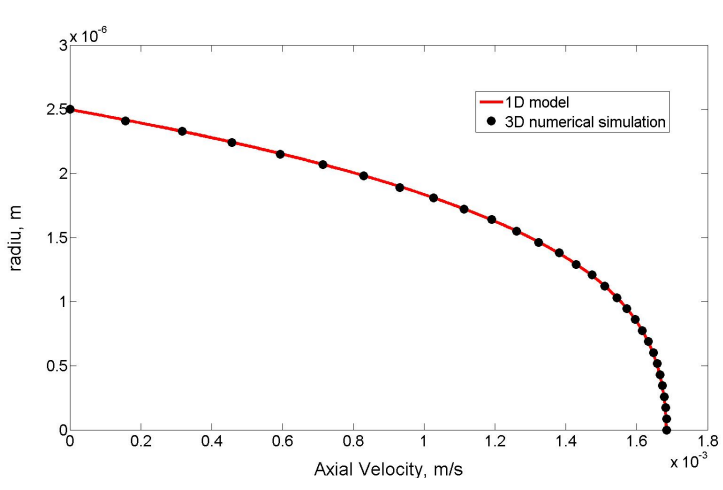

(a) Velocity profiles in a single pore

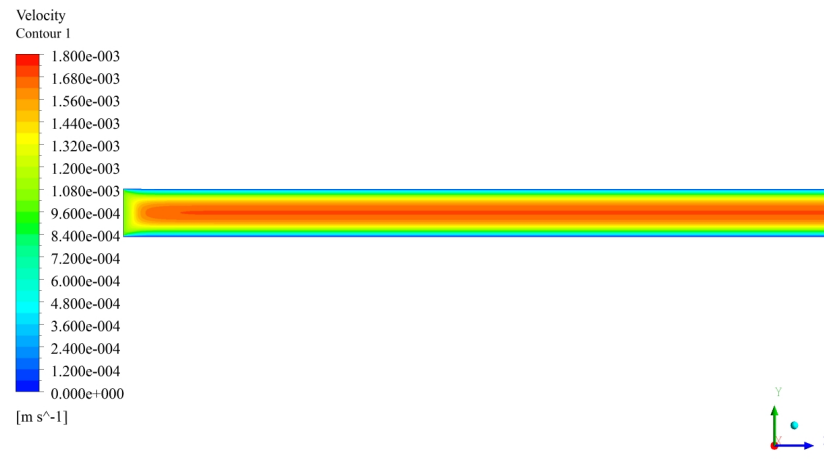

(b) Velocity contours in a mid-plane passing through pipe center line

Figure 1: Validation of the 1D model at $\frac{d p *}{d z *}=3.5617 \times 10^{7} \mathrm{~Pa} / \mathrm{m}$ for pipe diameter $d^{*}=5 \mu \mathrm{m}$

Eq (9) has been solved iteratively to investigate the laminar flow of generalized Newtonian fluid in circular pipes of different sizes. Velocity profiles and volume flow rate are determined at any pressure gradient for shear thinning as well as shear thickening fluids. Based on the solution, fanning friction factor is calculated at different pressure drop values using, 


$$
f=-\frac{d p^{*}}{d z^{*}} \frac{2 d^{*}}{\rho \bar{W}^{2}},
$$

where, $\bar{W}$ is the average flow velocity at the applied pressure gradient $\frac{d p^{*}}{d z^{*}}$ and $d^{*}$ is the pipe diameter in meters. Friction coefficient in pipe flow is usually plotted and/or determined as a function of $R e=\frac{\rho \bar{W} d^{*}}{\mu}$. It is observed in the Moody chart and in other models ( [20]) that $\mu$ does not depend on shear rate. For generalized Newtonian fluids, Dodge and Metzner [6] and Metzner [13] relied on power law to estimate the effective viscosity in the calculation of Reynolds number. Three different pipes diameters are used $(0.05 \mathrm{~m}, 0.005 \mathrm{~m}$ and $0.0005 \mathrm{~m})$ which have the same length to diameter ratio $\left(\frac{L^{*}}{d^{*}}=15.0\right)$. These sizes represent typical pore diameters ranges in a porous medium. In Eq (8) two parameters $\lambda$ and $n$ are varied to fit viscosity profiles of different polymeric solutions [4] where the values of zero-shear rate viscosity $\mu_{o}$, power law index $n$ and time constant $\lambda$ depend on the polymer concentration. In our calculations, the values used for the Carreau parameters are $\lambda=0.01$ and $n=1$ for Newtonian, $n=0.5$ for shear thinning and $n=1.5$ for shear thickening fluids. The zeroshear rate viscosity is set as $\mu_{o}=50.0$ Pa.s. Different values for $\lambda$ have been used also to investigate the transitional behavior of the flow from the Newtonian to the non-Newtonian region

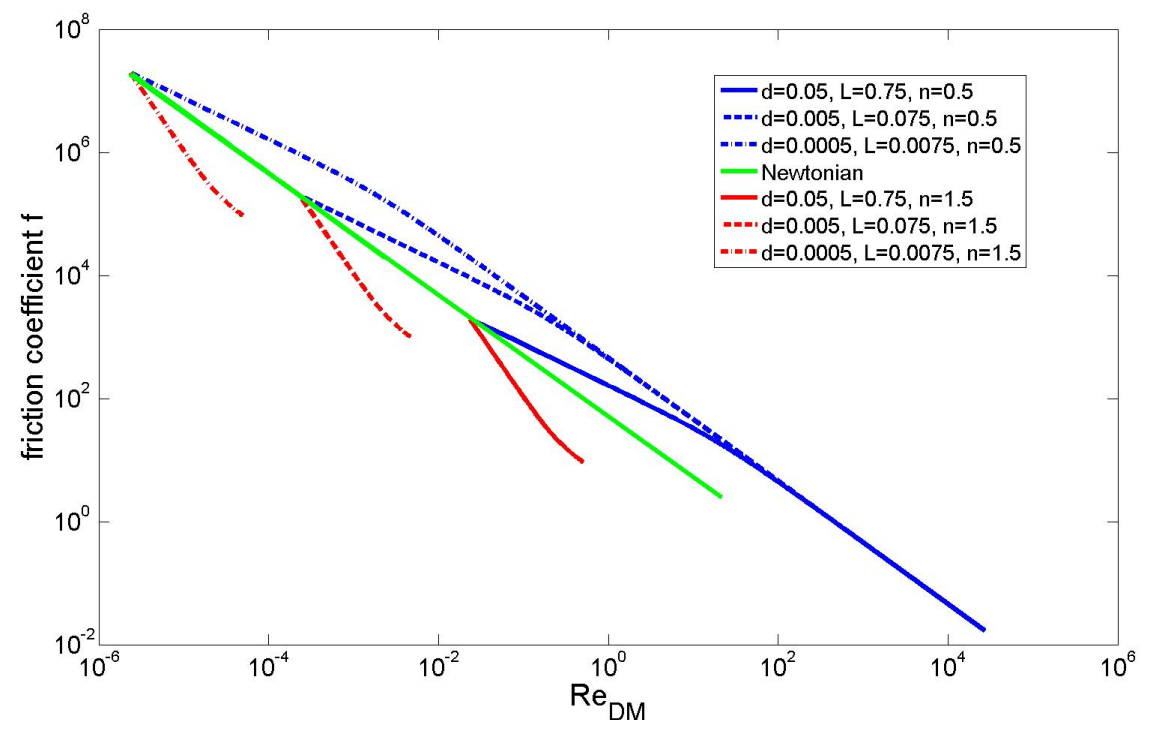

Figure 2: Friction coefficient versus Reynolds no. defined by Dodge and Metzner [6]

To study the variation of friction coefficient with Reynolds number given by (1), we need to determine the corresponding power law consistency factors of the above Carreau parameters. These parameters have been used to plot viscosity profiles of these carreau fluids. The power law consistency coefficient has been determined from these plots such that the viscosity profiles coincide with these of Carreau fluids in the power law regions such that Carreau and power law model represent the same generalized Newtonian fluids. Friction coefficient 
versus generalized Reynolds number $R e_{D M}$ is depicted in Figure 2. Friction coefficient of the shear thinning fluids varies in a fashion similar to the Newtonian fluids for $R e_{D M} \geq 1.0$. It is observed that by plotting friction coefficient versus generalized $R e_{D M}$, the Newtonian behavior of the flow at low Reynolds number can not be visualized. Instead this figure presents a remarkably different picture and seems quite counter intuitive. One would expect that the lines for non-Newtonian and Newtonian fluids merge or follow same slope at lower values of $R e_{D M}$ and at higher values differences may start to appear. However, the friction coefficient of shear thickening fluids behaves differently compared to the Newtonian fluids at low values of $R e_{D M}$. While at $R e_{D M}>1.0$, the slope of the line for the shear thinning fluids is same as that of the Newtonian fluids (green line). Therefore another definition of Reynolds number is needed to present the correct behavior of friction factor.

From the above non-dimensionlization of (2) it can be noticed that the reference velocity $w_{o}$, as given by (4), is an explicit function of pressure gradient. Here a new characteristic velocity $\overline{W_{o}}=\frac{w_{o}}{2}$ is proposed for the new definition of Reynolds number. The characteristic velocity $\overline{W_{o}}$ is equal to the mean velocity in circular pipe for the case of Newtonian fluid flow. However, it is different than the mean flow velocity for the flow of non-Newtonian fluid flow.

The Reynolds number $R e=\frac{\rho \overline{W_{o}} D^{*}}{\mu_{o}}$ can be written in terms of pressure gradient as

$$
R e=-\frac{d p^{*}}{d z^{*}} \frac{\rho a^{3}}{4 \mu_{o}^{2}}
$$

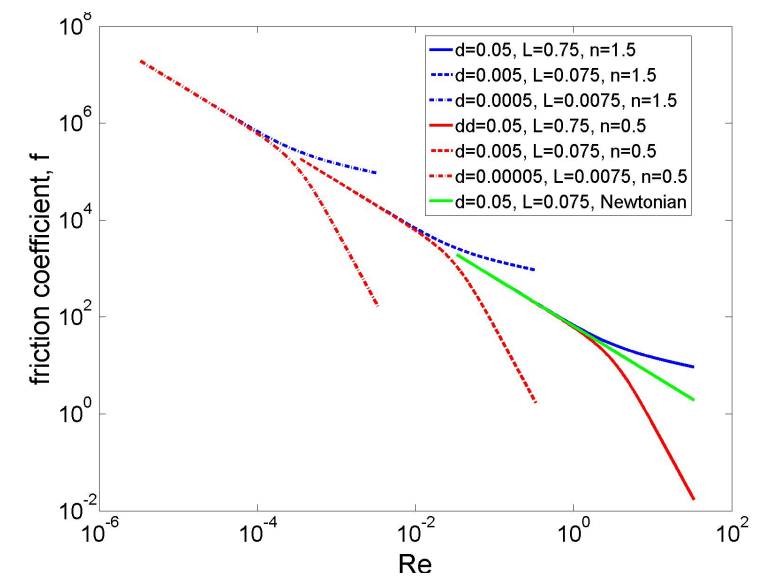

(a) Friction coefficient versus modified Reynolds no.

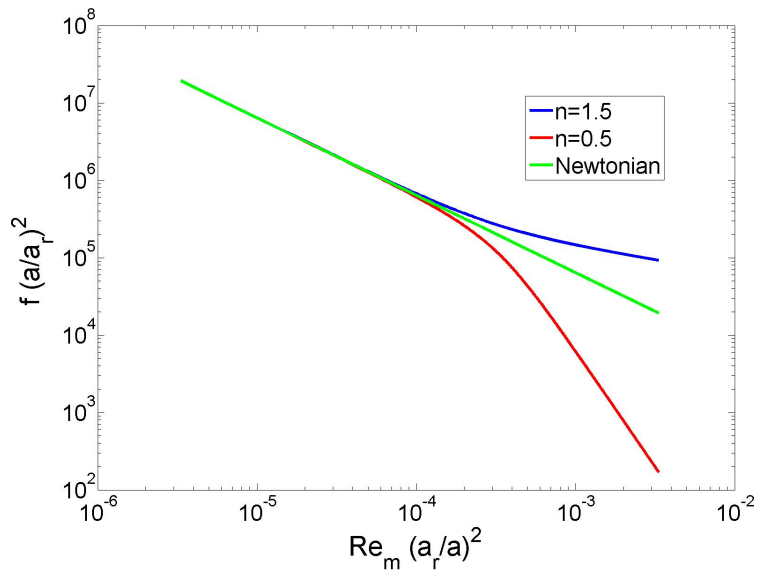

(b) Normalized friction coefficient versus modified Reynolds no.

Figure 3: Variation of friction coefficient versus modified Re

The calculated friction coefficient is now plotted versus the Reynolds number for three types of fluids $(n=0.5, n=1.0$ and $n=1.5)$ in each pipe as shown by Figure 3a. The calculations of flow parameters have been performed in three different pipes (i.e three different radii) of the same length to radius ratio $\frac{L}{a}=15$. For the same pressure drop, the 
reference velocity can be scaled linearly with the pipe radius as given by (4). In (4), the pipe radius $a$ has a power of 2 but the pressure gradient is scaled by a factor of $1 / a$ that results in scaling factor for the reference velocity is the pipe radius $a$. From the definition of Reynolds number in (13), the scaling factor is $\left(a_{r} / a\right)^{2}$, where $a_{r}=0.0005 m$. From equation (12), the scaling factor of the Friction coefficient is $\left(a / a_{r}\right)^{2}$. All the curves presented in 3a can be merged into a single curve (for $n=0.5, n=1.0$ or $n=1.5$ ) by using these scaling factors as shown in Figure 3b that provide a general closed form solution for any pore size. This figure shows that the friction coefficient of Newtonian fluids $(n=1)$ has a slope equal to -1 as known for Newtonian fluids. Friction coefficients of the shear thinning $(n=0.5)$ and shear thickening $(n=1.5)$ are also shown in this figure. This figure shows the Newtonian behavior at low Reynolds number where the curves of non-Newtonian fluids coincides with that of the Newtonian fluid at low Reynolds number. The non-Newtonian behavior at high Reynolds number is captured also where the curves of the shear thinning and shear thickening fluids deviate from that of the Newtonian curve. The plots of figure $3 \mathrm{~b}$ can be scaled to different pore throat radii using these scaling factors and used to determine the pressure drop due to certain flows rates in a pore-network model or in a bundle of tube model. For the assumed generalized Newtonian fluids, it is found that the viscosity is nearly constant and equal to the zero-shear rate viscosity $\mu_{o}$ up to Reynolds number $R e \leq 10^{-4}$. This observation is confirmed by Figures 5a-6b. The viscosity is almost constant along the radius and velocity profiles resembles a parabolic shape as seen in the case of Newtonian fluids.

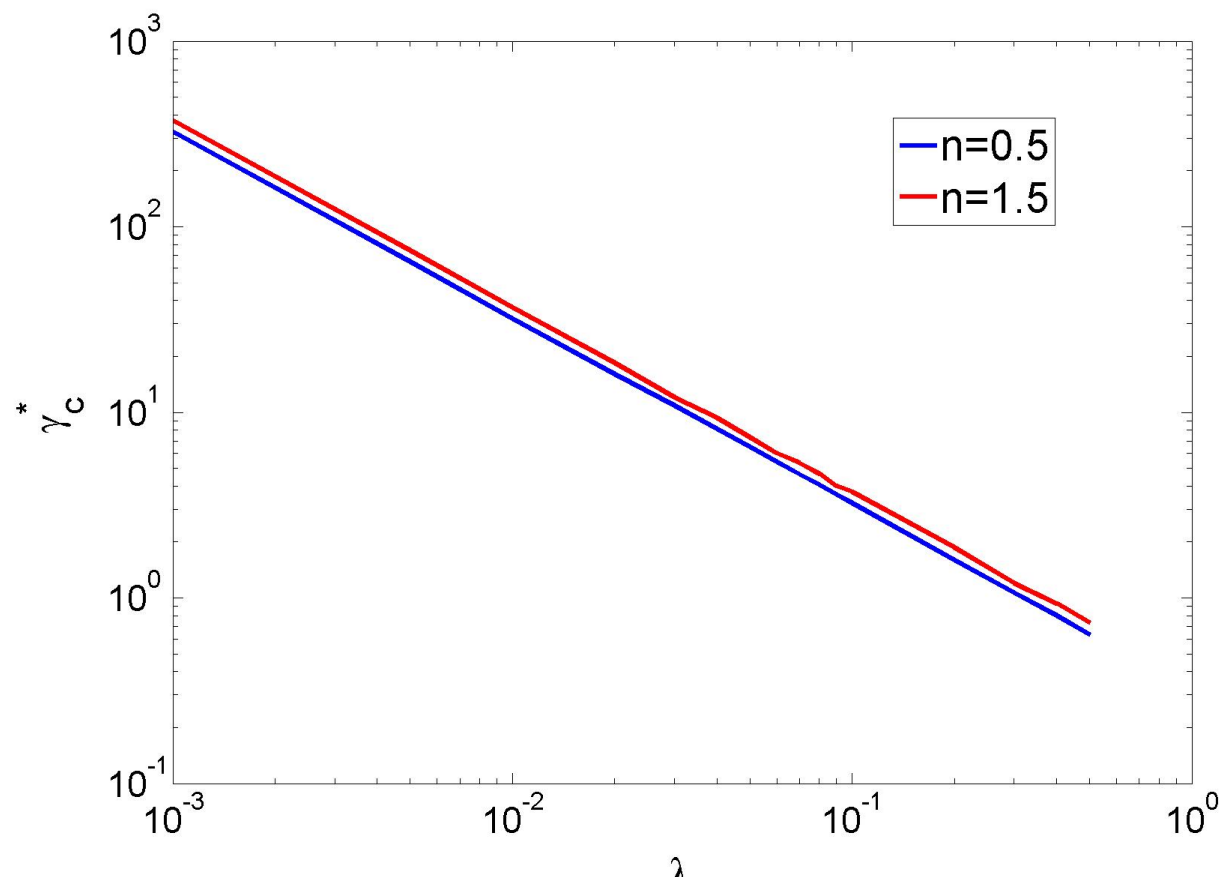

Figure 4: Critical reference shear strain rate variation with $\lambda$

As noticed that the non-Newtonian fluids exhibit 'pseudo-Newtonian' behavior at low shear rates. At certain critical shear rate, flow of a generalized Newtonian fluid deviates from the Newtonian behavior. The deviation from the pseudo-Newtonian to the non-Newtonian 
nature can be captured when the apparent viscosity at the pipe wall changes by $10 \%$ of the zero-shear rate viscosity. The reference shear strain rate is defined as the ratio of the reference velocity to the pipe radius $\gamma^{*}=\frac{w_{o}}{a}$. The critical reference shear strain rate is the reference strain rate at which flow changes its behavior from Newtonian to non-Newtonian. An expression for the critical reference shear strain rate is

$$
\gamma_{c}^{*}=-\frac{a}{4 \mu_{o}}\left(\frac{d p^{*}}{d z^{*}}\right)_{c},
$$

where $\left(\frac{d p^{*}}{d z^{*}}\right)_{c}$ is the critical pressure gradient.

It is observed that the deviation from the pseudo-Newtonian behavior to the non-Newtonian behavior based on $\gamma^{*}$ depends on the fitting parameter $\lambda$ used in the Carreau model. The variation of the $\gamma_{c}^{*}$ with the fitting parameter $\lambda$ is depicted in Figure 4 for shear thinning fluid as well as shear thickening fluid. The differences between the two curves in this figure are noticeable. The advantage of this figure is to separate the pseudo-Newtonian flow regime (below both curves) from the non-Newtonian flow regime (above both curves). It is imperative to mention the significance of $\lambda$ factor in Carreau model compared to power law model. The pseudo-Newtonian nature of generalized non-Newtonian fluids can be seen using Carreau model only. Moreover the segregation of Newtonian and non-Newtonian regimes based on $\lambda$ and $\gamma^{*}$ can be useful in modeling the behavior of generalized Newtonian fluid flows in porous media.

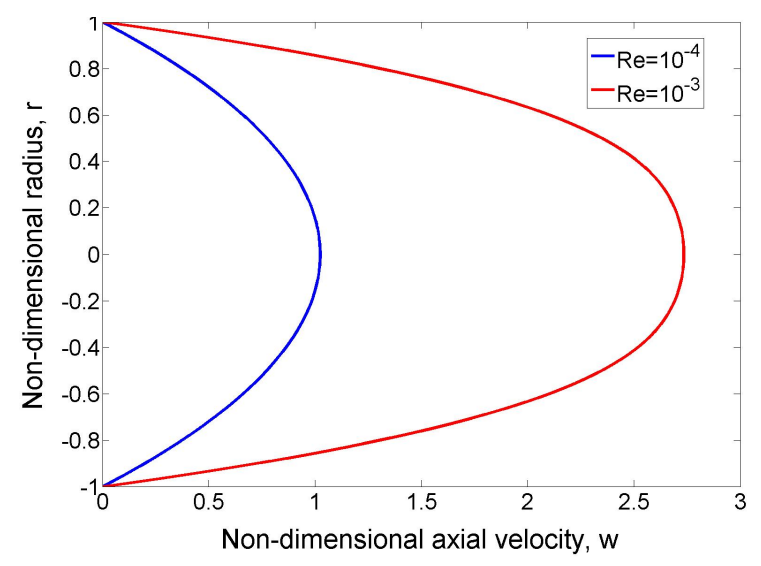

(a) Normalized velocity profiles

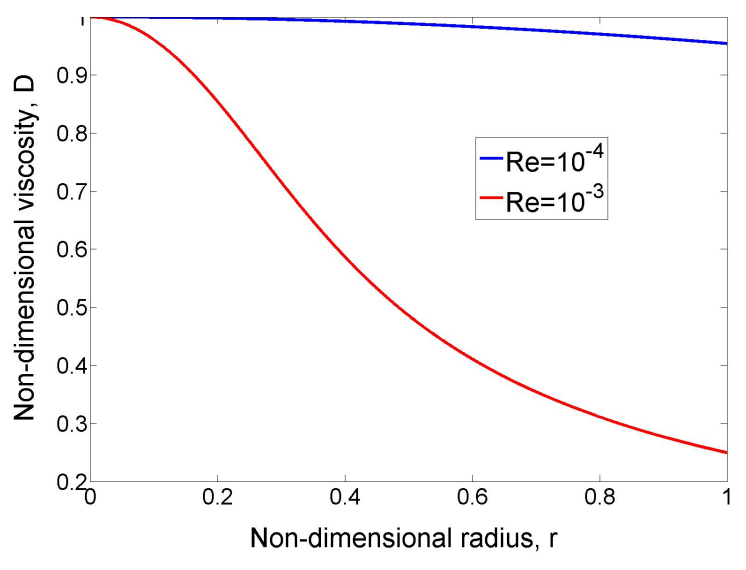

(b) Variation of apparant viscosity $\mu$ with radius

Figure 5: Velocity and apparent viscosity profiles for $n=0.5$ 


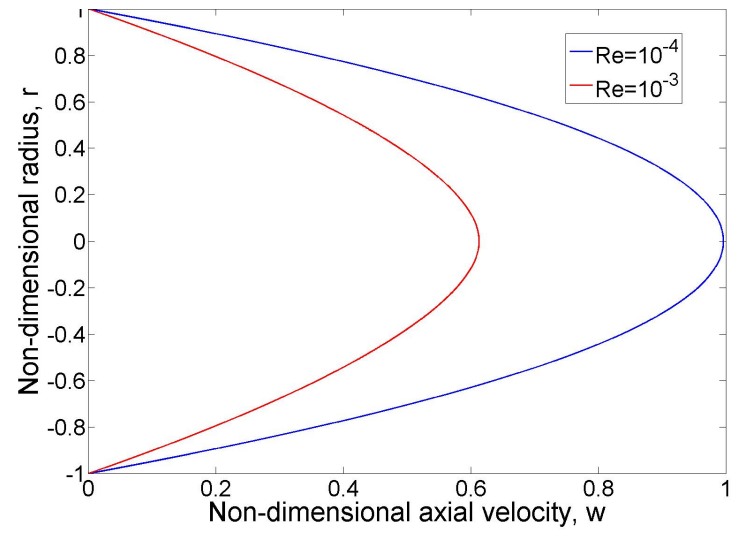

(a) Normalized velocity profiles

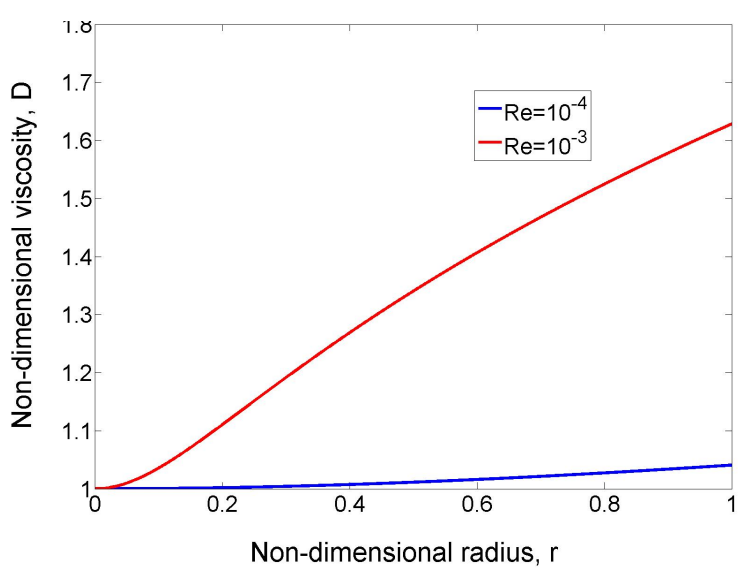

(b) Variation of apparant viscosity $\mu$ with radius

Figure 6: Velocity and apparent viscosity profiles for $n=1.5$

At higher Reynolds number, viscosity of the generalized Newtonian fluids is changing with the radius because of the high shear rates as depicted in Figures 5a-6b (blue curves). And it corresponds to the deviation of friction coefficient from the Newtonian case in Figure $3 \mathrm{~b}$. Friction coefficient for the case of shear thinning $(n=0.5)$ is much less that that of the Newtonian case because of lower values of viscosity at the pipe walls. While friction coefficient for the case of shear thickening $(n=1.5)$ is higher than that of the Newtonian case due to higher viscosity at the pipe walls.

It is useful also to plot the variation of friction coefficient versus the reference shear stain rate $\gamma^{*}$. Friction factors for $(n=0.5$ and $n=1.5)$ are plotted against $\gamma^{*}$ as shown in figure $7 \mathrm{a}$ for the three different pipe diameters. The flow of the non-Newtonian fluids behaves similar to that of Newtonian fluids up to a certain reference shear rate, for instance $\gamma^{*}=32.0$ for $\lambda=0.01$. A further increase in $\gamma^{*}$ shall lead to completely non-Newtonian behavior of flow. This compliments the findings presented earlier that the variation of flow behavior from pseudo-Newtonian to fully non-Newtonian depends on the critical value of $\gamma^{*}$, which depends on the value of $\lambda$. A single curve can be deduced here by scaling the friction coefficient using the area ratio $A / A_{r}$ regardless of pipe diameter. 


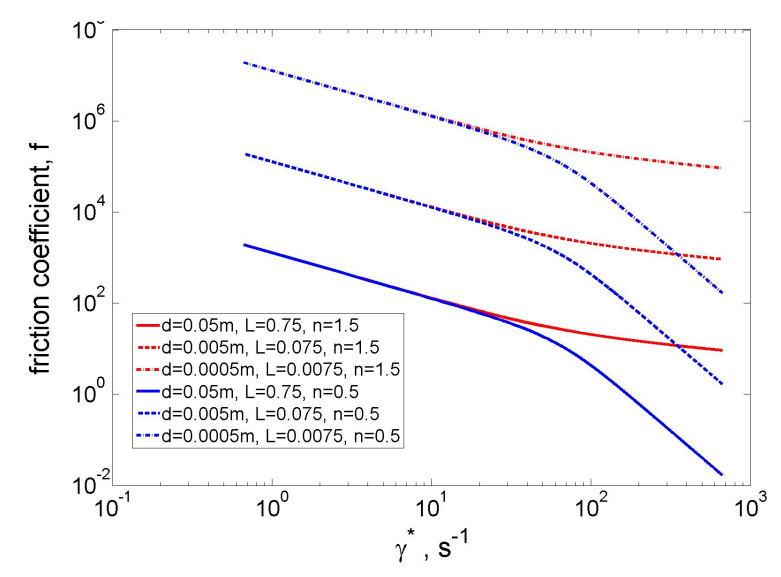

(a) Friction coefficient versus $\gamma^{*}$

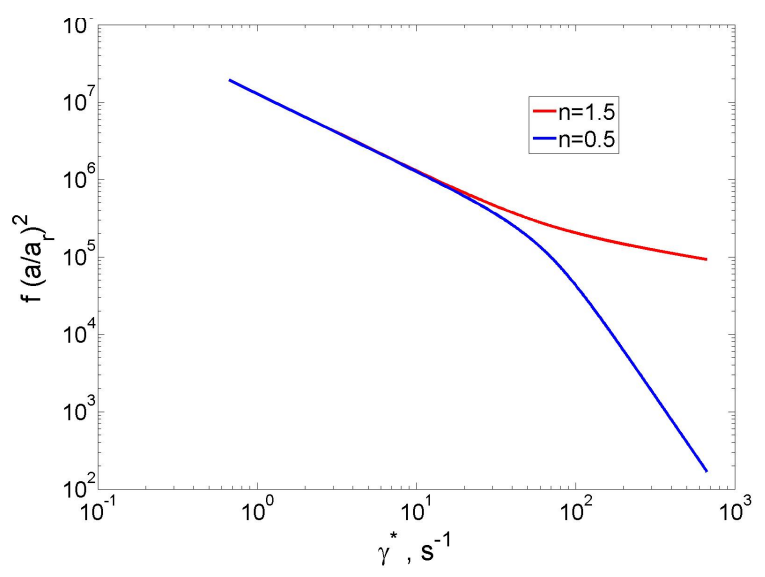

(b) Normalized friction coefficient versus $\gamma^{*}$

Figure 7: Variation of friction coefficient versus $\gamma^{*}$

\section{Conclusion}

Fully developed flow of generalized Newtonian fluids (shear thinning and shear thickening) in a circular pipes on the order of pore scales has been investigated. Friction factor has been plotted against a new definition of Reynolds based on the pressure gradient. Flow of generalized Newtonian fluids in circular pipes behave like Newtonian ones up to a certain Reynolds number where very low variation of viscosity along pipe radius is observed $(\mu \cong$ $\left.\mu_{o}\right)$. At higher Reynolds number, viscosity varies from the zero-shear rate to its lowest and/or highest values on the pipe wall for shear thinning and thickening fluids respectively. Therefore, lower friction coefficient for the shear thinning than that of Newtonian fluids is observed and higher friction coefficient for the shear thickening fluids. The paper provides

a simple graphical relationship to determine friction coefficient versus pressure gradient of generalized Newtonian fluid flows in circular capillaries. This relationship allows for more accurate calculation of pressure drop due to certain flow rate in a bundle of tubes or porenetwork models that represent a particular porous medium. 


\section{References}

[1] M. T. Balhoff and K. E. Thompson. Amacroscopic model for shear-thinning flowin packed beds based on network modeling. Chemical Engineering Science, 61:698-719, 2006.

[2] R.B. Bird, W.E. Stewart, and W. E. Lightfoot. Transport Phenomena. Wiley, New York, 2nd edition, 2002.

[3] M.J. Blunt. Flow in porous media-pore-network models and multiphase flow. Colloid Interface Sci., 6:197-207, 2001.

[4] F. Chenlo, R. Moreira, and C. Silva. Rheological properties od aqueous dispersions of tragacanth and guar gums at different concentration. Texture studies, 41:396-415, 2010.

[5] R. P. Chhabra. Bubbles, Drops, and Particles in Non-Newtonian Fluids. Taylor \& Francis Group, New York, 2007.

[6] D. W. Dodge and A. B. Metzner. turbulent flow of non-newtonian systems. A.I.CH. E., 5 No. 2:189-204, 1959.

[7] V. Di Federico, M. Pinelli, and R. Ugarelli. Estimates of effective permeability for nonnewtonian fluid flow in randomly heterogeneous porous media. Stochastic Env. Research and Risk Assessment, 24 No. 7:1067-1076, 2010.

[8] E. J. Garcia and J. F. Steffe. Comparison of friction factor equations for non-newtonian fluids in pipe flow. Food proc. Eng., 9:93-120, 1986.

[9] B. Guzel, I. Frigaarda, and D.M. Martinez. Predicting laminar-turbulent transition in poiseuille pipe flow for non-newtonian fluids. Chem. Eng. Sc., 64:254-264, 2009.

[10] S. Liu and J. Masliyah. On non-newtonian fluid flow in ducts and porous media. Chem. Eng. Sci., 53 No. 6:1175-1201, 1998.

[11] S. Liu and J. Masliyah. Non-linear flows in porous media. J. Non-Newtonian Fluid Mech., 86:229-252, 1999.

[12] X. Lopez, P.H. Valvatne, and M. J. Blunt. Predictive network modeling of single-phase non-newtonian flow in porous media. Colloid and Interface Science, 264:256 - 265, 2003.

[13] A. B. Metzner. Non-newtonian fluid flow. Industrial and Eng. Chem., 49 NO. 9:14291432, 1957.

[14] A. F. Morais, H. Seybold, H. J. Herrmann, and J. Andrade. Non-newtonian fluid flow through three-dimensional disordered porous media. Phys. Rev. Lett., 103 No. 19:194502, 2009.

[15] M. Pakdemirli, P. Sari, and B. Solmaz. Analytical and numerical solutions of a generalized hyperbolic non-newtonian fluid flow. Z. Naturforsch, 65a:151-160, 2010. 
[16] C.L. Perrin, P. Tardy, K. Sorbie, and J. Crawshaw. Experimental and modeling study of newtonian and non-newtonian fluid flow in pore network micromodels. Colloid and Interface Science, 295 No. 2:542-550, 2006.

[17] F.T. Pinho and J.H. Whitela. Flow of non-newtonian fluids in a pipe. J. non-Newtonian Fluid Mech., 34:129-124, 1990.

[18] A. Raoof, H. S. Majid, and A. Leijnse. Upscaling transport of adsorbing solutes in porous media: pore-network modeling. Vadose Zone, 9:624-636, 2010.

[19] N. Sabiri and J. Comiti. Pressure drop in non-newtonian purely viscous fluid flow through porous media. Chem Eng Science, 50 No. 7:1193-1201, 1995.

[20] I. Shames. Mechanics of Fluids. McGraw Hill, New York, 1994.

[21] T. Sochi. Non-newtonian flow in porous media. Polymer, 51:5007-5023, 2010.

[22] S.P. Sullivan, L.F. Gladden, and M.L. Johns. Simulation of power-law fluid flow through porous media using lattice boltzmann techniques. Non-Newtonian Fluid Mech., 133 No. 2-3:91-98, 2006.

[23] G.H. Tang and Y.B. Lu. A resistance model for newtonian and power-law non-newtonian fluid transport in porous media. Transp Porous Med., 104:435-449, 2014.

[24] B. A. Toms. Detection of a wall effect in laminar flow of solutions of a linear polymer. Colloid Sci., 4:511-521, 1949.

[25] T. Tosco, D. L. Marchisio, F. Lince, and R. Sethi. Extension of the darcy-forchheimer law for shear-thinning fluids and validation via pore-scale flow simulations. Transp Porous Med, 96:1-20, 2013.

[26] C. D. Tsakiroglou. A methodology for the derivation of non-darcian models for the flow of generalized newtonian fluids in porous media. Non-Newtonian Fluid Mechanics, 105 No. 2-3:79-110, 2002.

[27] S. Woudberg, J.P. Du Plessis, and G.J.F. Smit. Non-newtonian purely viscous flow through isotropic granular porous media. Chem. Eng. Sci., 61 No. 13:4299-4308, 2006.

[28] L.S. Yao, Md. M. Molla, and S. G. Moulic. Fully-developed circular pipe flow of a non-newtonian pseudoplastic fluid. Universal J. Mechanical Eng., 1(2):23-31, 2013.

[29] M. Yun. Seepage characteristics study on power-law fluid in fractal porous media. Mathematical Problems Eng., 2014:813561, 2014. 